\title{
ANTICONVULSANT AND NEUROTOXICITY EVALUATION OF SOME NEWLY SYNTHESIZED THIAZOLYL COUMARIN DERIVATIVES
}

\author{
${ }^{1}$ M. Faiz Arshad, ${ }^{2}$ Nadeem Siddiqui, ${ }^{1,3}$ Ahmed Elkerdasy, \\ ${ }^{1}$ Abdulmohsen H. Al Rohaimi and ${ }^{2}$ Suroor A. Khan \\ ${ }^{1}$ Department of Pharmaceutical Sciences, College of Pharmacy, Shaqra University, Saudi Arabia \\ ${ }^{2}$ Department of Pharmaceutical Chemistry, Faculty of Pharmacy, \\ Jamia Hamdard (Hamdard University), New Delhi 110062, India \\ ${ }^{3}$ Department of Biochemistry, College of Veterinary Medicine, Sadat University, Egypt
}

Received 2014-02-27; Received 2014-03-08; Accepted 2014-03-26

\begin{abstract}
The present series of compounds were synthesized with the aim to develop newer anticonvulsant agents that are comparatively more efficacious and safer than the currently used anticonvulsant agents. Various thiazolyl coumarins were synthesized by the reaction of 3-(bromoacetyl)-2H-chromen-2-one with different substituted aryl thiourea. The structures of the synthesized compounds were confirmed by spectral data and elemental analyses. Compounds were tested for anticonvulsant activity utilizing Pen Tylenetetra Zoleinduced seizure (PTZ) and Maximal Electroshock Seizure (MES) tests at 30, 100 and $300 \mathrm{mg} \mathrm{kg}^{-1} \mathrm{dose}^{-}$ level. Neurotoxicity and ethanol potentiation test of the compounds were also assessed at the same dose level. Two compounds of the series $3 \mathrm{~g}$ and $3 \mathrm{j}$ exhibited significant anticonvulsant activity at $30 \mathrm{mg} \mathrm{kg}^{-1}$ dose level with lesser neurotoxicity than the standard drug phenytoin.
\end{abstract}

Keywords: Coumarin, Thiazole, Anticonvulsant Activity, Neurotoxicity

\section{INTRODUCTION}

Epilepsy is one of the most common of the serious neurological disorders (Abuhamed et al., 2008) that affect $1-2 \%$ people in all nations and of all races. In epilepsy, the normal pattern of neuronal activity becomes disturbed, causing strange sensations, emotions and behavior or sometimes convulsions, muscle spasms and loss of consciousness. These physical changes are called epileptic seizures. These seizures are transient signs and/or symptoms due to abnormal, excessive or synchronous neuronal activity in the brain (Fisher et al., 2006). Over $30 \%$ of people with epilepsy do not have seizure control even with the best available medications (Talevi et al., 2006).

The earlier literatures exposed some plant coumarins possessing moderate anticonvulsant activity
(Chaturvedi et al., 1974; Singh et al., 2006). In addition many thiazoles and fused thiazoles displayed anticonvulsant activity (Bachir et al., 1990; Sarro et al., 1993) and therefore connecting both the moieties viz. Coumarin and thiazole expected to have the synergistic effect in dealing with the epilepsy.

The present study focuses on to synthesize a series of 3-(2-arylamino-1, 3-thiazol-4-yl)-2-chromanones (3a-p) and screen them for anticonvulsant activity using Maximal Electroshock Seizure (MES) and subcutaneous Pen Tylenetetra Zole (scPTZ) induced seizure models.

\section{MATERIALS AND METHODS}

\subsection{Chemistry}

Melting points were taken in open capillary tubes and are uncorrected. ${ }^{1} \mathrm{H}$ NMR spectra were recorded on a $\begin{array}{ll}\text { Corresponding Author: } & \begin{array}{l}\text { M. Faiz Arshad, Department of Pharmaceutical Sciences, Col } \\ \text { dawadmi, P.O. Box 33, Saudi Arabia Tel: } 00966594082153\end{array}\end{array}$ 
Bruker model DRX 300 NMR spectrometer in DMSO- $d_{6}$ using Tetra Methyl Silane (TMS) as an internal standard. IR spectra were recorded on BIO-RAD FTS 135 spectrometer using $\mathrm{KBr}$ pellet. The elemental analyses $(\mathrm{C}, \mathrm{H}, \mathrm{N})$ were performed using Perkin-Elmer model $240 \mathrm{C}$ analyzer and all values were within $\pm 0.4 \%$ of the theoretical compositions. The $\log \mathrm{P}$ values for all the synthesized compounds were calculated using ACD lab version 8.0. TLC was carried out using silica gel G. All the chemicals and solvents used were obtained from merck.

\subsection{Synthesis of the Titled Compounds (3a-p)}

\subsubsection{3-Acetyl-2H-Chromen-2-one (1)}

A mixture of salicyaldehyde $(0.5 \mathrm{moL})$ and ethyl acetoacetate $(0.5 \mathrm{moL})$ was stirred and cooled. To this mixture $10 \mathrm{~g}$ of piperidine was added with shaking. The mixture was maintained at freezing temperature for $2-3 \mathrm{~h}$ resulting in a yellow colored solid mass, which was separated out. It was recrystallized from ethanol to get the target compound.

\subsection{3-(Bromoacetyl)-2H-Chromen-2-One (2)}

To a solution of compound (1) $(0.25 \mathrm{moL})$ in $200 \mathrm{~mL}$ of alcohol free chloroform, bromine $(0.25 \mathrm{moL})$ in 25 $\mathrm{mL}$ of chloroform was added with intermittent shaking. The mixture was warmed to decompose an addition product. The mixture was heated for $15 \mathrm{~min}$, cooled and filtered to get a solid mass which on washing with ether gave the desired product. It was recrystallized from acetic acid to give colorless needles.

\subsection{3-(2-Arylamino-1, Chromanone (3a-p) \\ 3-Thiazol-4-yl)-2-}

A suspension of compound (2) (0.1 moL) in 175 $\mathrm{mL}$ of hot ethanol was heated with arylthiourea $(0.1$ moL), giving a clear solution that soon deposited as crystals. It was filtered, washed with ethanol and then boiled with water containing sodium acetate which yielded the target compound. The product obtained was recrystallized with ethanol.

\subsection{Pharmacology}

\subsubsection{Anticonvulsant Activity}

The investigations were conducted on swiss albino mice of either sex (25-30 g). Food and water were withdrawn prior to the experiments. All the compounds (3a-p) were dissolved in propylene glycol. Initially all compounds were administered ip at doses of 30, 100300 $\mathrm{mg} \mathrm{kg}^{-1}$ to mice. Activity was established using the MES and scPTZ tests according to the protocol (Krall et al., 1978; Clark et al., 1984) by Antiepileptic Drug Development Program (ADD), Epilepsy Branch, National Institute of Health, Bethesda, MD, USA.

\subsection{Maximal Electroshock Seizure (MES) Test}

Mice were prescreened $24 \mathrm{~h}$ before by delivering maximal electroshock $50 \mathrm{~mA} ; 60 \mathrm{~Hz}$ and $0.2 \mathrm{~s}$ duration by means of corneal electrodes. A drop of $0.9 \%$ sodium chloride was instilled in each eye prior to the application of electrodes in order to prevent death of the animal. Abolition of hind limb tonic extensor component of the seizure in half or more of the animals is defined as protection.

\subsection{Subcutaneous Pen Tylenetetra Zol Test (scPTZ)}

The scPTZ test utilized a dose of pentylenetetrazol 70 $\mathrm{mg} \mathrm{kg}$. This produced clonic seizures lasting for a period of at least $5 \mathrm{sec}$. The test compounds administered at the three graded doses i.e., 30, 100 and $300 \mathrm{mg} \mathrm{kg}^{-1}$ i.p. At the anticipated time the convulsant was administered subcutaneously. Animals were observed over a 30 min period. Absence of clonic spasm in half or more of the animals in the observed time period indicated a compound's ability to abolish the effect of pentylenetetrazol on seizure threshold.

\subsection{Neurotoxicity Studies}

\subsubsection{Rotorod Test}

The minimal motor impairment was measured in mice by the rotorod test (Dunham and Miya, 1957). The mice were trained to stay on an accelerating rotorod of diameter $3.2 \mathrm{~cm}$ that rotates at $10 \mathrm{rpm}$. Trained animals were given ip injection of the test compounds in doses of 30,100 and $300 \mathrm{mg} \mathrm{kg}^{-1}$. Neurotoxicity was indicated by the inability of the animal to maintain equilibrium on the rod for at least one minute in each of the three trials.

\subsection{Ethanol Potentiation Test}

Mice were treated with the test compound and $1 \mathrm{~h}$ later with ethanol $2.5 \mathrm{~g} \mathrm{~kg}^{-1}$ ip. This dose of ethanol did not induce lateral position in the control animals. The number of animals that were in the lateral position after receiving ethanol in each group was determined (Clerici and Pocar, 2001).

\subsection{In Silico Studies}

The in silico studies were performed by using software Chem3D Pro 6.0 version. 


\section{RESULTS}

\subsection{Chemistry}

The compounds (3a-p) were synthesized according to the scheme shown in Fig. 1. The synthesis of 3-acetyl-2Hchromen-2-one (1) was involved a reaction between salicylaldehyde and ethylacetoacetate in presence of piperidine. In the second step bromination of the compound (1) yielded 3-(bromoacetyl)-2H-chromen-2one (2) which on cyclization in presence of aryl thiourea formed 3-(2-arylamino-1, 3-thiazol-4-yl)-2-chromanone (3a-p). The physical parameters of all synthesized compounds are given in Table 1. All the synthesized compounds were well characterized by elemental analysis and spectroscopic data. In IR spectra absorption bands for $\mathrm{N}-\mathrm{H}, \mathrm{C}-\mathrm{H}, \mathrm{C}=\mathrm{O}$ and $\mathrm{C}=\mathrm{N}$ were found in the region of 3588-3311, 3073-2866, 1761-1669 and 1598-1550 $\mathrm{cm}^{-1}$ respectively. The ${ }^{1} \mathrm{H}$ NMR spectra showed two distinct aromatic zones of multiplet at $\delta$ values $6.65-7.93 \mathrm{ppm}$. A broad singlet assigned for $\mathrm{N}-\mathrm{H}$ attached with thiazole. The C-4, C-5 protons of coumarin and one thiazole proton were distinct and characteristic. The spectral data of the compounds (3a-p) are placed in Table 2.<smiles>CCOC(=O)CC(C)=O</smiles><smiles></smiles>

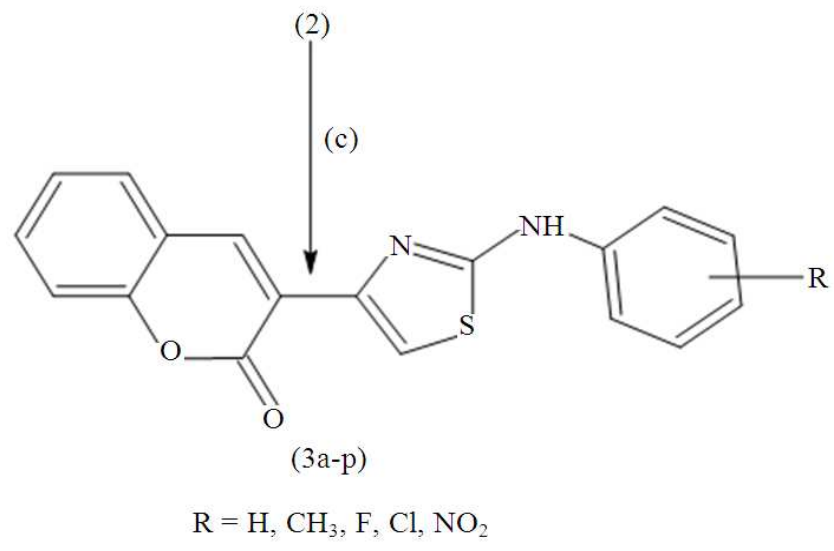

Fig. 1. Reagents and conditions; (a) Piperidine, Ethanol (b) $\mathrm{Br}_{2}, \mathrm{CHCl}_{3}$, Heat (c) Substituted arylthiourea, Heat. 
Table 1. Physical characterization data of the compounds (3a-p)

\begin{tabular}{lllllll}
\hline Compound & $\mathrm{R}$ & $\mathrm{Mol}$. formula & \%Yield & $\mathrm{Mp}{ }^{\circ} \mathrm{C}$ & ${ }^{\mathrm{a}} \mathrm{R}_{\mathrm{f}}$ & ${ }^{\mathrm{b}} \mathrm{Log} P$ \\
\hline $3 \mathrm{a}$ & $\mathrm{H}$ & $\mathrm{C}_{18} \mathrm{H}_{12} \mathrm{~N}_{2} \mathrm{O}_{2} \mathrm{~S}$ & 71 & 154 & 0.81 & 4.62 \\
$3 \mathrm{~b}$ & $2-\mathrm{CH}_{3}$ & $\mathrm{C}_{19} \mathrm{H}_{14} \mathrm{~N}_{2} \mathrm{O}_{2} \mathrm{~S}$ & 64 & 162 & 0.82 & 5.09 \\
$3 \mathrm{c}$ & $3-\mathrm{CH}_{3}$ & $\mathrm{C}_{19} \mathrm{H}_{14} \mathrm{~N}_{2} \mathrm{O}_{2} \mathrm{~S}$ & 65 & 158 & 0.78 & 5.09 \\
$3 \mathrm{~d}$ & $4-\mathrm{CH}_{3}$ & $\mathrm{C}_{19} \mathrm{H}_{14} \mathrm{~N}_{2} \mathrm{O}_{2} \mathrm{~S}$ & 58 & 169 & 0.79 & 5.09 \\
$3 \mathrm{e}$ & $2-\mathrm{OCH}_{3}$ & $\mathrm{C}_{19} \mathrm{H}_{14} \mathrm{~N}_{2} \mathrm{O}_{3} \mathrm{~S}$ & 66 & 141 & 0.81 & 4.52 \\
$3 \mathrm{f}$ & $3-\mathrm{OCH}_{3}$ & $\mathrm{C}_{19} \mathrm{H}_{14} \mathrm{~N}_{2} \mathrm{O}_{3} \mathrm{~S}$ & 71 & 173 & 0.72 & 4.88 \\
$3 \mathrm{~g}$ & $4-\mathrm{OCH}_{3}$ & $\mathrm{C}_{1} \mathrm{H}_{14} \mathrm{~N}_{2} \mathrm{O}_{3} \mathrm{~S}$ & 59 & 175 & 0.74 & 4.57 \\
$3 \mathrm{~h}$ & $2-\mathrm{F}$ & $\mathrm{C}_{18} \mathrm{H}_{11} \mathrm{FN}_{2} \mathrm{O}_{2} \mathrm{~S}$ & 63 & 161 & 0.55 & 4.59 \\
$3 \mathrm{i}$ & $3-\mathrm{F}$ & $\mathrm{C}_{18} \mathrm{H}_{11} \mathrm{FN}_{2} \mathrm{O}_{2} \mathrm{~S}$ & 56 & 123 & 0.81 & 5.11 \\
$3 \mathrm{j}$ & $4-\mathrm{F}$ & $\mathrm{C}_{18} \mathrm{H}_{11} \mathrm{FN}_{2} \mathrm{O}_{2} \mathrm{~S}$ & 69 & 157 & 0.76 & 5.07 \\
$3 \mathrm{k}$ & $2-\mathrm{Cl}$ & $\mathrm{C}_{18} \mathrm{H}_{12} \mathrm{ClN}_{2} \mathrm{O}_{2} \mathrm{~S}$ & 77 & 141 & 0.78 & 5.13 \\
$3 \mathrm{l}$ & $3-\mathrm{Cl}$ & $\mathrm{C}_{18} \mathrm{H}_{12} \mathrm{ClN}_{2} \mathrm{O}_{2} \mathrm{~S}$ & 68 & 175 & 0.66 & 5.66 \\
$3 \mathrm{~m}$ & $4-\mathrm{Cl}$ & $\mathrm{C}_{18} \mathrm{H}_{12} \mathrm{ClN}_{2} \mathrm{O}_{2} \mathrm{~S}$ & 68 & 151 & 0.68 & 5.62 \\
$3 \mathrm{n}$ & $2-\mathrm{NO}_{2}$ & $\mathrm{C}_{18} \mathrm{H}_{11} \mathrm{~N}_{3} \mathrm{O}_{4} \mathrm{~S}$ & 63 & 149 & 0.73 & 4.88 \\
$3 \mathrm{o}$ & $3-\mathrm{NO}_{2}$ & $\mathrm{C}_{18} \mathrm{H}_{11} \mathrm{~N}_{3} \mathrm{O}_{4} \mathrm{~S}$ & 60 & 123 & 0.87 & 5.01 \\
$3 \mathrm{p}$ & $4-\mathrm{NO}_{2}$ & $\mathrm{C}_{18} \mathrm{H}_{11} \mathrm{~N}_{3} \mathrm{O}_{4} \mathrm{~S}$ & 74 & 165 & 0.85 & 5.08 \\
\hline
\end{tabular}

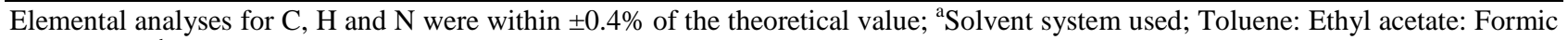
acid (5:4:1); ${ }^{\mathrm{b}} C \log P$ was calculated using ACD lab version 8.0

Table 2. Spectral data of synthesized compounds (3a-p)

\begin{tabular}{|c|c|c|}
\hline Compound & $\mathrm{IR}(\mathrm{KBr}) \mathrm{cm}^{-1}$ & ${ }^{1} \mathrm{H}$ NMR $\left(\mathrm{DMSO}-d_{6}\right) \delta \mathrm{ppm}$ \\
\hline $3 \mathrm{a}$ & $3588(\mathrm{~N}-\mathrm{H}), 1713(\mathrm{C}=\mathrm{O}, \alpha$ pyrone $), 1594(\mathrm{C}=\mathrm{N})$ & $\begin{array}{l}\text { 6.74-7.01 (m, 5H, Ar-H), 7.06 (bs, } 1 \mathrm{H}, \mathrm{NH}), 7.23-7.50(\mathrm{~m}, 3 \mathrm{H}, \text { coum. }) \\
\text { 7.67-7.69 (d, 1H, H-5 coum.), 8.13 (s, } 1 \mathrm{H}, \text { thiazol.), } 8.28 \text { (s, 1H, H-4 coum) }\end{array}$ \\
\hline $3 b$ & $\begin{array}{l}3447(\mathrm{~N}-\mathrm{H}), 3044(\mathrm{C}-\mathrm{H}), 1663 \\
(\mathrm{C}=\mathrm{O}, \alpha \text { pyrone }), 1563(\mathrm{C}=\mathrm{N})\end{array}$ & $\begin{array}{l}2.07\left(\mathrm{~s}, 3 \mathrm{H}, \mathrm{CH}_{3}\right), 6.67-7.05(\mathrm{~m}, 5 \mathrm{H}, \mathrm{Ar}-\mathrm{H} \& \mathrm{NH}), 7.17-7.56(\mathrm{~m}, 3 \mathrm{H}, \text { coum. }) \\
7.69-7.71(\mathrm{~d}, 1 \mathrm{H}, \mathrm{H}-5 \text { coum. }), 8.19(\mathrm{~s}, 1 \mathrm{H}, \text { thiazol. }), 8.25(\mathrm{~s}, 1 \mathrm{H}, \mathrm{H}-4 \text { coum })\end{array}$ \\
\hline $3 \mathrm{c}$ & $\begin{array}{l}3552(\mathrm{~N}-\mathrm{H}), 2928(\mathrm{C}-\mathrm{H}), 1709(\mathrm{C}=\mathrm{O}, \alpha \text { pyrone }), \\
1556(\mathrm{C}=\mathrm{N})\end{array}$ & $\begin{array}{l}2.15\left(\mathrm{~s}, 3 \mathrm{H}, \mathrm{CH}_{3}\right), 6.61(\mathrm{~s}, 1 \mathrm{H}, \mathrm{Ar}-\mathrm{H}), 6.67-6.88(\mathrm{~m}, 3 \mathrm{H}, \mathrm{Ar}-\mathrm{H}), 6.98 \\
(\mathrm{~s}, 1 \mathrm{H}, \mathrm{NH}), 7.21-7.50(\mathrm{~m}, 3 \mathrm{H}, \text { coum. }), 7.70-7.72(\mathrm{~d}, 1 \mathrm{H}, \mathrm{H}-5 \text { coum. }) \\
8.13(\mathrm{~s}, 1 \mathrm{H}, \text { thiazol. }), 8.32(\mathrm{~s}, 1 \mathrm{H}, \mathrm{H}-4 \text { coum. })\end{array}$ \\
\hline $3 \mathrm{~d}$ & $\begin{array}{l}3504(\mathrm{~N}-\mathrm{H}), 2945(\mathrm{C}-\mathrm{H}), 1719(\mathrm{C}=\mathrm{O}, \alpha \text { pyrone }) \\
1573(\mathrm{C}=\mathrm{N})\end{array}$ & $\begin{array}{l}2.20\left(\mathrm{~s}, 3 \mathrm{H}, \mathrm{CH}_{3}\right), 7.05-7.17(\mathrm{~m}, 5 \mathrm{H}, \mathrm{Ar}-\mathrm{H} \& \mathrm{NH}), 7.26-7.61(\mathrm{~m}, 3 \mathrm{H}, \text { coum. }), \\
7.71-7.73(\mathrm{~d}, 1 \mathrm{H}, \mathrm{H}-5 \mathrm{coum} .), 8.19(\mathrm{~s}, 1 \mathrm{H}, \text { thiazol.), } 8.24(\mathrm{~s}, 1 \mathrm{H}, \mathrm{H}-4 \text { coum. }\end{array}$ \\
\hline $3 \mathrm{e}$ & $\begin{array}{l}3449(\mathrm{~N}-\mathrm{H}), 3013(\mathrm{C}-\mathrm{H}), 1672(\mathrm{C}=\mathrm{O}, \alpha \text { pyrone }) \\
1588(\mathrm{C}=\mathrm{N})\end{array}$ & $\begin{array}{l}3.67\left(\mathrm{~s}, 3 \mathrm{H}, \mathrm{OCH}_{3}\right), 6.40-6.74(\mathrm{~m}, 4 \mathrm{H}, \mathrm{Ar}-\mathrm{H}), 7.35-7.63(\mathrm{~m}, 4 \mathrm{H}, \text { coum. \& } \mathrm{NH}) \\
7.63-7.65(\mathrm{~d}, 1 \mathrm{H}, \mathrm{H}-5 \mathrm{coum} .), 8.12(\mathrm{~s}, 1 \mathrm{H}, \text { thiazol. }), 8.29(\mathrm{~s}, 1 \mathrm{H}, \mathrm{H}-4 \mathrm{coum} .)\end{array}$ \\
\hline $3 \mathrm{f}$ & $\begin{array}{l}3436(\mathrm{~N}-\mathrm{H}), 3028(\mathrm{C}-\mathrm{H}), 1712(\mathrm{C}=\mathrm{O}, \alpha \text { pyrone }), \\
1579(\mathrm{C}=\mathrm{N})\end{array}$ & $\begin{array}{l}3.64\left(\mathrm{~s}, 3 \mathrm{H}, \mathrm{OCH}_{3}\right), 6.06(\mathrm{~s}, 1 \mathrm{H}, \mathrm{NH}), 6.30-6.71(\mathrm{~m}, 3 \mathrm{H}, \mathrm{Ar}-\mathrm{H}), 6.78 \\
(\mathrm{~s}, 1 \mathrm{H}, \mathrm{Ar}-\mathrm{H}), 7.16-7.47(\mathrm{~m}, 3 \mathrm{H}, \text { coum.), } 7.59-7.61(\mathrm{~d}, 1 \mathrm{H}, \mathrm{H}-5 \mathrm{coum} .) \\
8.19(\mathrm{~s}, 1 \mathrm{H}, \text { thiazol. }), 8.34(\mathrm{~s}, 1 \mathrm{H}, \mathrm{H}-4 \text { coum. }\end{array}$ \\
\hline $3 g$ & $\begin{array}{l}3448(\mathrm{~N}-\mathrm{H}), 3047(\mathrm{C}-\mathrm{H}), 1752(\mathrm{C}=\mathrm{O}, \alpha \text { pyrone }), \\
1583(\mathrm{C}=\mathrm{N})\end{array}$ & $\begin{array}{l}3.69\left(\mathrm{~s}, 3 \mathrm{H}, \mathrm{OCH}_{3}\right), 6.67-6.69(\mathrm{~d}, 2 \mathrm{H}, \mathrm{Ar}-\mathrm{H}), 6.74-6.76(\mathrm{~d}, 2 \mathrm{H}, \mathrm{Ar}-\mathrm{H}), 7.06 \\
\text { (bs, } 1 \mathrm{H}, \mathrm{NH}), 7.22-7.46(\mathrm{~m}, 3 \mathrm{H}, \text { coum. }) \text {, 7.67-7.69 (d, 1H, H-5 coum.), } \\
8.21(\mathrm{~s}, 1 \mathrm{H}, \text { thiazol. }), 8.32(\mathrm{~s}, 1 \mathrm{H}, \mathrm{H}-4 \text { coum. })\end{array}$ \\
\hline $3 \mathrm{~h}$ & $3433(\mathrm{~N}-\mathrm{H}), 1729(\mathrm{C}=\mathrm{O}, \alpha$ pyrone $), 1598(\mathrm{C}=\mathrm{N})$ & $\begin{array}{l}\text { 6.73-7.12 (m, 5H, Ar-H \& NH), 7.23-7.51 (m, } 3 \mathrm{H}, \text { coum.), } 7.53(\mathrm{~s}, 1 \mathrm{H} \text {, thiazol.), } \\
\text { 7.64-7.66 (d, } 1 \mathrm{H}, \mathrm{H}-5 \mathrm{coum} .), 8.20(\mathrm{~s}, 1 \mathrm{H} \text {, thiazol.), } 8.29(\mathrm{~s}, 1 \mathrm{H}, \mathrm{H}-4 \text { coum. })\end{array}$ \\
\hline $3 \mathrm{i}$ & $3468(\mathrm{~N}-\mathrm{H}), 1745(\mathrm{C}=\mathrm{O}, \alpha$ pyrone $), 1583(\mathrm{C}=\mathrm{N})$ & $\begin{array}{l}\text { 6.42-6.47 (m, 1H, Ar-H), 6.51-6.57 (dd, 1H, Ar-H), 6.88-6.90 (d, 1H, Ar-H), } \\
\text { 7.13-7.19 (m, 1H, Ar-H), 7.26-7.52 (m, 4H, coum. \& NH), 7.61-7.63 } \\
\text { (d, 1H, H-5 coum.), 8.11 (s, 1H, thiazol.), 8.33 (s, 1H, H-4 coum.) }\end{array}$ \\
\hline $3 \mathrm{j}$ & $3336(\mathrm{~N}-\mathrm{H}), 1737(\mathrm{C}=\mathrm{O}, \alpha$ pyrone $), 1582(\mathrm{C}=\mathrm{N})$ & $\begin{array}{l}\text { 6.74-6.76 (d, 2H, Ar-H), } 7.07 \text { (bs, 1H, NH), 7.13-7.15 (d, 2H, Ar-H), } \\
\text { 7.24-7.50 (m, 3H, coum.), } 7.71-7.73(\mathrm{~d}, 1 \mathrm{H}, \mathrm{H}-5 \text { coum. }), 8.16(\mathrm{~s}, 1 \mathrm{H} \text {, thiazol.), } \\
8.29(\mathrm{~s}, 1 \mathrm{H}, \mathrm{H}-4 \text { coum. }\end{array}$ \\
\hline $3 \mathrm{k}$ & $3451(\mathrm{~N}-\mathrm{H}), 1750(\mathrm{C}=\mathrm{O}, \alpha$ pyrone $), 1579(\mathrm{C}=\mathrm{N})$ & $\begin{array}{l}\text { 6.67-6.78 (m, 4H, Ar-H), 7.31-7.51 (m, 3H, coum.), 7.68-7.70 (d, 1H, H-5 } \\
\text { coum.), } 7.95(\mathrm{bs}, 1 \mathrm{H}, \mathrm{NH}), 8.23(\mathrm{~s}, 1 \mathrm{H}, \text { thiazol.), } 8.39(\mathrm{~s}, 1 \mathrm{H}, \mathrm{H}-4 \text { coum. }\end{array}$ \\
\hline 31 & $3464(\mathrm{~N}-\mathrm{H}), 1743(\mathrm{C}=\mathrm{O}, \alpha$ pyrone $), 1569(\mathrm{C}=\mathrm{N})$ & $\begin{array}{l}\text { 6.83-6.89 (m, 3H, Ar-H), } 7.31(\mathrm{~s}, 1 \mathrm{H}, \mathrm{Ar}-\mathrm{H}) 7.46-7.52(\mathrm{~m}, 3 \mathrm{H}, \text { coum. }) \\
7.58(\mathrm{bs}, 1 \mathrm{H}, \mathrm{NH}), 7.66-7.68(\mathrm{~d}, 1 \mathrm{H}, \mathrm{H}-5 \mathrm{coum} .), 8.09(\mathrm{~s}, 1 \mathrm{H} \text {, thiazol.), } \\
8.31(\mathrm{~s}, 1 \mathrm{H}, \mathrm{H}-4 \text { coum. })\end{array}$ \\
\hline $3 \mathrm{~m}$ & $3519(\mathrm{~N}-\mathrm{H}), 1726(\mathrm{C}=\mathrm{O}, \alpha$ pyrone $), 1574(\mathrm{C}=\mathrm{N})$ & $\begin{array}{l}\text { 6.94-6.97 (d, 2H, Ar-H), } 7.09(\mathrm{bs}, 1 \mathrm{H}, \mathrm{NH}), 7.18-7.20(\mathrm{~d}, 1 \mathrm{H}, \mathrm{Ar}-\mathrm{H}) 7.22-7.49 \\
(\mathrm{~m}, 3 \mathrm{H}, \text { coum.), } 7.59-7.61(\mathrm{~d}, 1 \mathrm{H}, \mathrm{H}-5 \mathrm{coum} .), 8.11(\mathrm{~s}, 1 \mathrm{H}, \text { thiazol.), } 8.29 \\
(\mathrm{s}, 1 \mathrm{H}, \mathrm{H}-4 \text { coum. })\end{array}$ \\
\hline $3 \mathrm{n}$ & $3531(\mathrm{~N}-\mathrm{H}), 1738(\mathrm{C}=\mathrm{O}, \alpha$ pyrone $), 1563(\mathrm{C}=\mathrm{N})$ & $\begin{array}{l}\text { 6.91-6.95 (t, 1H, Ar-H), 7.23-7.27 (m, 3H, Ar-H), 7.37-7.41 (m, 1H, Ar-H), } \\
\text { 7.46 (bs, 1H, NH), 7.46-7.53 (m, 3H, coum.), 7.63-7.65 (d, 1H, H-5 coum.), } \\
\text { 7.83-7.85 (d, 1H, Ar-H), 8.14-8.16 (d, 1H, Ar-H), } 8.07 \text { (s, 1H, thiazol.), } \\
8.39(\mathrm{~s}, 1 \mathrm{H}, \mathrm{H}-4 \text { coum.) }\end{array}$ \\
\hline 30 & $3524(\mathrm{~N}-\mathrm{H}), 1729(\mathrm{C}=\mathrm{O}, \alpha$ pyrone $), 1550(\mathrm{C}=\mathrm{N})$ & 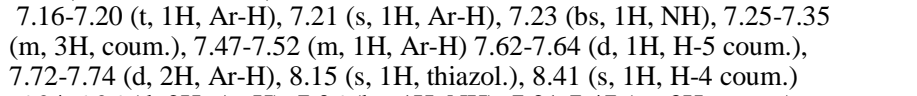 \\
\hline $3 p$ & $3498(\mathrm{~N}-\mathrm{H}), 1748(\mathrm{C}=\mathrm{O}, \alpha$ pyrone $), 1565(\mathrm{C}=\mathrm{N})$ & $\begin{array}{l}\text { 6.94-6.96 (d, 2H, Ar-H), } 7.06 \text { (bs, 1H, NH), } 7.21-7.47 \text { (m, 3H, coum.), } \\
\text { 7.69-7.71 (d, 1H, H-5 coum.), 8.03-8.05 (d, 2H, Ar-H), } 8.19 \text { (s, 1H, thiazol.), } \\
8.38(\mathrm{~s}, 1 \mathrm{H}, \mathrm{H}-4 \text { coum. })\end{array}$ \\
\hline
\end{tabular}


Table 3. Anticonvulsant and neurotoxicity data of compounds (3a-p)

\begin{tabular}{|c|c|c|c|c|c|c|c|}
\hline \multirow[b]{2}{*}{ Compound } & \multicolumn{2}{|l|}{ MES } & \multicolumn{2}{|c|}{ scPTZ } & \multicolumn{2}{|c|}{ Neurotoxicity } & \multirow{2}{*}{$\begin{array}{l}\text { Ethanol } \\
\text { potentiation }\end{array}$} \\
\hline & $0.5 \mathrm{~h}$ & $4 \mathrm{~h}$ & $0.5 \mathrm{~h}$ & $4 \mathrm{~h}$ & $0.5 \mathrm{~h}$ & $4 \mathrm{~h}$ & \\
\hline $3 a$ & 100 & 300 & 300 & $(-)$ & 300 & $(-)$ & 300 \\
\hline $3 b$ & $(-)$ & $(-)$ & $X$ & $\mathrm{X}$ & $\mathrm{X}$ & $\mathrm{X}$ & $\mathrm{X}$ \\
\hline $3 c$ & 100 & 300 & 300 & $(-)$ & 100 & 300 & 100 \\
\hline $3 d$ & 100 & 300 & 100 & $(-)$ & $(-)$ & $(-)$ & $(-)$ \\
\hline $3 e$ & 300 & $(-)$ & $\mathrm{X}$ & $\mathrm{X}$ & $\mathrm{X}$ & $\mathrm{X}$ & $\mathrm{X}$ \\
\hline $3 \mathrm{f}$ & $(-)$ & $(-)$ & $X$ & $X$ & $\mathrm{X}$ & $\mathrm{X}$ & $X$ \\
\hline $3 g$ & 30 & 300 & $(-)$ & $(-)$ & $(-)$ & $(-)$ & $(-)$ \\
\hline $3 \mathrm{~h}$ & 300 & $(-)$ & $X$ & $\mathrm{X}$ & $\mathrm{X}$ & $X$ & $\mathrm{X}$ \\
\hline $3 \mathrm{i}$ & 300 & 300 & $\mathrm{X}$ & $\mathrm{X}$ & $\mathrm{X}$ & $\mathrm{X}$ & $\mathrm{X}$ \\
\hline $3 \mathrm{j}$ & 30 & 100 & 300 & $(-)$ & 300 & $(-)$ & 300 \\
\hline $3 \mathrm{k}$ & $(-)$ & 300 & $X$ & $\mathrm{X}$ & $\mathrm{X}$ & $\mathrm{X}$ & $X$ \\
\hline 31 & 300 & $(-)$ & $X$ & $X$ & $\mathrm{X}$ & $X$ & $X$ \\
\hline $3 \mathrm{~m}$ & 100 & $(-)$ & 300 & 300 & 100 & $(-)$ & $(-)$ \\
\hline $3 n$ & 300 & 300 & $X$ & $\mathrm{X}$ & $X$ & $\mathrm{X}$ & $\mathrm{X}$ \\
\hline 30 & 300 & $(-)$ & $\mathrm{X}$ & $\mathrm{X}$ & $\mathrm{X}$ & $\mathrm{X}$ & $\mathrm{X}$ \\
\hline $3 p$ & 100 & $(-)$ & 100 & 300 & $(-)$ & 300 & $(-)$ \\
\hline Phenytoin ${ }^{\mathrm{a}}$ & 30 & 30 & $(-)$ & $(-)$ & 100 & 100 & $\mathrm{X}$ \\
\hline
\end{tabular}

Dose of 30,100 and $300 \mathrm{mg} \mathrm{kg}^{-1}$ were administered i.p. The figures indicate the minimum dose whereby bioactivity was demonstrated in half or more mice. The (-) indicates an absence of activity at maximum dose administered $\left(300 \mathrm{mg} \mathrm{kg}^{-1}\right)$. The (X) indicates not tested. ${ }^{\mathrm{a}}$ Data from reference (White et al., 1995)

\subsection{Anticonvulsant Activity}

Anticonvulsant evaluation of compounds (3a-p) in mice utilizing MES and scPTZ models are summarized in Table 3 together with the neurotoxicity data. Among the tested compounds of the series, $3 \mathrm{~d}, 3 \mathrm{~g}, 3 \mathrm{j}$ and $3 \mathrm{p}$ showed highest activity against MES test. The other compounds $3 \mathrm{a}, 3 \mathrm{c}$ and $3 \mathrm{~m}$ exhibited moderately active in the MES test. Some compounds e.g., 3e, 3h, 3i, 31, 3n and 30 were least active while rest compounds were inactive.

Only those compounds were chosen for chemoshock activity utilizing scPTZ animal model that showed highest to moderate activity against MES test. Therefore compounds $3 \mathrm{a}, 3 \mathrm{c}, 3 \mathrm{~d}, 3 \mathrm{~g}, 3 \mathrm{j}, 3 \mathrm{~m}$ and $3 \mathrm{p}$ were subjected against scPTZ test. Compounds $3 \mathrm{~d}$ and $3 \mathrm{p}$ showed significant activity while compounds $3 \mathrm{a}, 3 \mathrm{c}, 3 \mathrm{j}$ and $3 \mathrm{~m}$ exhibited least activity. Although compound $3 \mathrm{~g}$ that showed highly active against MES test, found devoid of activity against scPTZ test.

The undesired side effect i.e., neurotoxicity of highly and moderately active compounds were evaluated by conducting the rotorod and ethanol potentiation tests and the results are expressed in Table 3. Compounds $3 \mathrm{c}$ and $3 \mathrm{~m}$ that exhibited encouraging anti-MES activity were found to have considerable neurotoxicity. Two anticonvusant active compounds, $3 \mathrm{a}$ and $3 \mathrm{j}$ showed moderately toxic in both rotorod and ethanol potentiation tests. Compounds $3 \mathrm{~d}, 3 \mathrm{~g}, 3 \mathrm{~m}$ and $3 \mathrm{p}$ successfully passed the rotorod and ethanol potentiation test.

The majority of the active compounds exhibited $C \log$ $P$ (Calculated $\log P$ ) values ranging from 5.07-5.62 except compounds $3 \mathrm{a}(C \log P=4.62)$ and $3 \mathrm{~g}(C \log P=4.57)$.

\section{DISCUSSION}

\subsection{Structure Activity Relationship}

In general, the para substituted derivatives were found to be most active of the series in agreement with the previously published work (Siddiqui et al., 2009a). The unsubstituted derivative showed activity in both the seizure models and almost similar results were discussed in the previous data (Dawood et al., 2006). Substitutions with electron releasing groups $\left(-\mathrm{CH}_{3},-\mathrm{OCH}_{3}\right)$ further increased the activity and the result was found in agreement with the previously published results (Siddiqui et al., 2009b). The size of the group seemed to govern the scPTZ activity as the derivatives with larger methoxy group were found to be inactive in scPTZ screen whereas derivatives substituted with smaller methyl group favors both anti-MES as well as anti-scPTZ activity. Substitutions with electron withdrawing groups $\left(\mathrm{F}, \mathrm{Cl}, \mathrm{NO}_{2}\right)$ resulted in further enhancements of the anticonvulsant activity and the similar results were found in the earlier literature (Kumar et al., 2013). The sizes of the group also played an important role as the derivatives substituted with fluoro group were most active followed by chloro and nitro. It was also seen that the substitutions with larger functional groups $\left(-\mathrm{OCH}_{3}, \mathrm{Cl}, \mathrm{NO}_{2}\right)$ showed minimal or absence of activity at $4 \mathrm{~h}$ indicative of the quick onset and short duration of action of the compounds. 


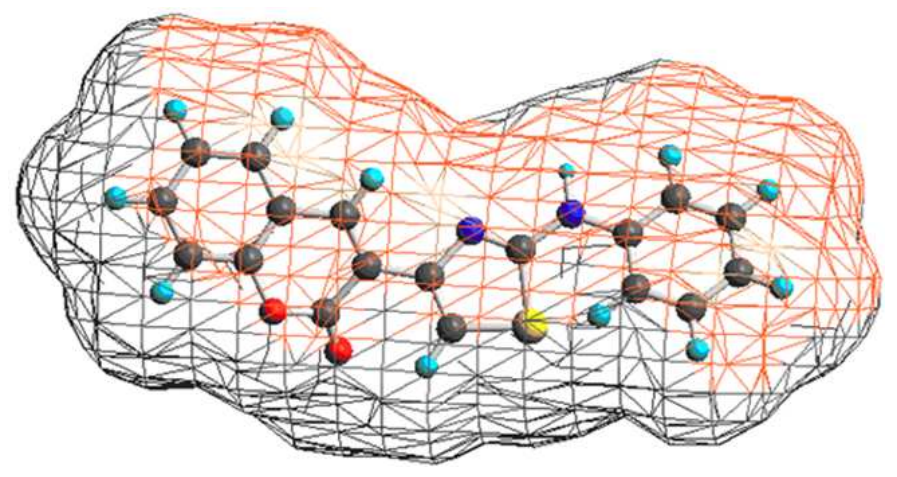

Fig. 2. The three-dimensional optimized structure of 3-[2-(phenylamino)-1, 3-thiazol-4-yl]-2H-chromen-2-one 3a) showing solvent accessible surfaces.

Interestingly, all the para substituted derivatives were found to show potent anticonvulsant activity and similar results could be found in the previous work (Aggarwal et al., 2009). This may be due to the fact that the para substituted derivatives are better fitted in the pocket at the receptor site.

\subsection{In Silico Studies}

The in silico studies were performed on the compound 3-[2-(phenylamino)-1, 3-thiazol-4-yl]-2Hchromen-2-one (3a) which represents the general prototype structure of the synthesized compounds. It was observed that the structure had solvent accessible surfaces all over it (Fig. 2), which may have contributed to the high lipophilicity of the titled compounds and the results are comparable with the previous literature (Siddiqui and Ahsan, 2009).

\section{CONCLUSION}

The idiopathic nature of epilepsy is the major hurdle to establish a specific target in developing antiepileptic drugs. The present study was aimed to develop new antiepileptic drugs based on structural modifications. The results of the investigations suggest that two compounds i.e., $3 \mathrm{~g}$ and $3 \mathrm{j}$ of the newly synthesized series of thiazolyl coumarins displayed significant anticonvulsant activity with lesser neurotoxicity than the standard drug phenytoin. These compounds can be considered as lead molecules for future investigations.

\section{ACKNOWLEDGEMENT}

Researchers are thankful to the University Grants Commission (UGC), New Delhi for providing financial assistance to conduct this project. Authors also wish to thank biochemistry laboratory, College of Veterinary Medicine, Sadat University, Egypt for conducting some experiments on animals. The various support of Shaqra University is highly acknowledged.

\section{REFERENCES}

Abuhamed, M., B. Xiao, K. Xia, Y. Fang and L. Lili, 2008. Voltage-Gated channels as causative agents for epilepsies. Am. J. Immunol., 4: 43-50. DOI: 10.3844/ajisp.2008.43.50

Aggarwal, N., P. Mishra, B.P. Nagori, R. Aggarwal and J. Jain, 2009. Anticonvulsant and neurotoxicity evaluation of some N(4) phenyl substituted pyridyl semicarbazones. Central Nervous Syst. Agents Medicinal Chem., 9: 295-299. PMID: 20021362

Bachir, M., J.P. Riffaud, J. Lacolle, J. Lemoine and A.D. Almeida et al., 1990. Synthesis and anticonvulsant activity of some 2-(N-substituted glycylamino)-4methylthiazoles. Eur. J. Med. Chem., 25: 71-74. DOI: 10.1016/0223-5234(90)90167-2

Chaturvedi, A.K., S.S. Parmar, S.C. Bhatnagar, G. Misra and S.K. Nigam et al., 1974. Anticonvulsant and antiinflammatory activity of natural plant coumarins and triterpenoids. Res. Commun. Chem. Pathol. Pharmacol., 9: 11-22. PMID: 4438821

Clark, C.R., M.J.M. Wells, R.T. Sansom, G.N. Norris and R.C. Dockens et al., 1984. Anticonvulsant activity of some 4-aminobenzamides. J. Med. Chem., 27: 779-782. DOI: 10.1021/jm00372a013

Clerici, F. and D. Pocar, 2001. Synthesis of 2-amino-5sulfanyl-1, 3, 4-thiadiazole derivatives and evaluation of their antidepressant and anxiolytic activity. J. Med. Chem., 44: 931-936. DOI: $10.1021 / \mathrm{jm} 001027 \mathrm{w}$ 
Dawood, K. M.., H.A. Gawad, E.A. Rageb, M. Ellithey and H.A. Mohamed, 2006. Synthesis, anticonvulsant and anti-inflammatory evaluation of some new benzotriazole and benzofuran-based heterocycles. Bioorgicn Med. Chem., 14: 3672-3680. DOI: 10.1016/j.bmc.2006.01.033

Sarro, D.G.B., M. Zapalla, S. Grasso, A. Chimirri and A. De Sarro et al., 1993. Molecular requirement for anticonvulsant activity in a series of thiazolo-1,4benzodiazepine derivatives and comparison with classical benzodiazepines. General Pharmac. Vascular Syst., 24: 877-884. DOI: 10.1016/03063623(93)90163-R

Dunham, N.W. and T.A. Miya, 1957. A note on a simple apparatus for detecting neurological deficit in rats and mice. J. Am. Pharm. Assoc. Sci., 46: 208-209. DOI: $10.1002 / j p s .3030460322$

Fisher, R., W. Van Emde Boas and W. Blume, 2006. Treatment of new onset seizures: Predicting longterm outcome. Epilepsia, 46: 470-472. DOI: 10.1111/j.1535-7511.2006.00139.x

Krall, R.L., J.K. Penry, B.G. White, H.J. Kupferberg and E.A. Swinyard, 1978. Antiepileptic drug development: II. Anticonvulsant drug screening. Epilepsia, 19: 409-428. DOI: 10.1111/j.15281157.1978.tb04507.x

Kumar, D, V.K. Sharma, R. Kumar, T. Singh and H. Singh et al., 2013. Design, synthesis and anticonvulsant activity of some new 5,7-dibromoisatin semicarbazone derivatives. EXCLI J., 12: 628-64.
Siddiqui, N., A. Rana, S.A. Khan, S.E. Haque and M.F. Arshad et al., 2009a. Synthesis and preliminary screening of benzothiazol-2-yl thiadiazole derivatives for anticonvulsant activity. Acta Pharmaceutical, 59: 441-451. DOI: 10.2478/v10007009-0031-X

Siddiqui, N., P. Alam and W. Ahsan, 2009b. Design, synthesis and in-vivo pharmacological screening of N-3-(substituted diphenyl)-5-phenyl-1H-pyrazoline1- carbothioamide derivatives. Arch. Pharmazie Chem. Life Sci., 342: 173-181. DOI: 10.1002/ardp.200800130. PMID: 19194967

Siddiqui, N. and W. Ahsan, 2009. Benzothiazole incorporated barbituric acid derivatives: Synthesis and anticonvulsant screening. Arch. Pharmazie Chem. Life Sci., 342: 462-468. DOI: 10.1002/ardp.200900002. PMID: 19565603

Singh, D., M. Rawat, G. Garg, S. Saraf and S. Saraf et al., 2006. Artemisia scoparia: A review. Pharmacogonsy Magazine, 2: 27-30.

Talevi, A., J.J. Prieto, L.E. Bruno-Blanch and E. Castro, 2006. On application of carhart atom pairs to predict anticonvulsant activity. Am. J. Biochem. Biotechnol., 2: 119-128.

White, H.S., J.H. Woodhead, M.R. Franklin, E.A. Swinyard and H.A. Wolf et al., 1995. General Principles. In: Experimental Selection, Quantification and Evaluation of Antiepileptic Drugs. Levy, R.H., R.H. Mattson and B.S. Meldrum, (Eds.)., Antiepileptic Drugs, Raven Press, New York, USA, pp: 99-110. 